\title{
Effects of Neurokinin-1 Receptor Inhibition on Anxiety Behavior in Neonatal Rats Selectively Bred for an Infantile Affective Trait
}

\author{
Amanda L. Schott, Betty Zimmerberg* \\ Department of Psychology and Program in Neuroscience, Williams College, Williamstown, USA \\ Email: ${ }^{*}$ bzimmerb@williams.edu
}

Received 1 July 2014; revised 27 July 2014; accepted 4 August 2014

Copyright (C) 2014 by authors and Scientific Research Publishing Inc.

This work is licensed under the Creative Commons Attribution International License (CC BY). http://creativecommons.org/licenses/by/4.0/

(c) (i) Open Access

\begin{abstract}
Interest in understanding the etiology and developing new treatments for anxiety disorders in children and adolescents has led to recent studies of neurotransmitters not traditionally associated with neural pathways for fear and anxiety. The binding of the neurotransmitter substance $P$ (SP) to its neurokinin-1 (NK1) receptor may be a crucial component in mediating the anxiety response. While previous studies using rodent models have documented the anxiolytic effects of SP antagonists, the role of individual differences in affective temperament has not yet been examined in studies of drug response. This study used intracerebroventricular injections of the NK1 antagonist Spantide II at concentrations of 10 and $100 \mathrm{pmol}$ to examine the consequences of blocking the SP-NK1 pathway in high and low line rats selectively bred for high or low levels of ultrasonic distress calls after a brief maternal separation. Affective temperament was a significant factor in determining drug response. Spantide II resulted in a significant reduction of distress calls in subjects in the high anxiety line, while low line subjects with low anxiety were resistant to the drug. These data indicate that the SP-NK1 pathway could be an important therapeutic target for the treatment of various stress disorders, but drug response might be influenced by the individual's state anxiety or history of chronic stress.
\end{abstract}

\section{Keywords}

Anxiety, Substance P, Neurokinin Receptor, Spantide, Ultrasonic Vocalizations

\footnotetext{
*Corresponding author.
} 


\section{Introduction}

Anxiety disorders are among the most prevalent mental health disorders, including social phobia, post-traumatic stress disorder, obsessive-compulsive disorder, and panic disorder. Globally, estimates of the prevalence of anxiety disorders range from 5.3\% in African cultures to 10.4\% in Euro/Anglo cultures [1]. Anxiety behaviors are affective reactions that use neural pathways that evolved for mammalian fear responses. Fear responses can be observed very early in life; for example, the normative response of separation anxiety appears in infants at around 18 months of age and can persist until about three years of age. Recently, attention has focused on better detection and developing new treatments for anxiety disorders in adolescents and children. In a national survey of American teenagers, anxiety symptoms were reported by $31.9 \%$ of the respondents, with $8.3 \%$ meeting anxiety diagnostic criteria of severe impairment and/or distress [2]. The median age for disorder onset was also earlier for anxiety disorders (6 years of age) than for other mental health illnesses. Anxiety disorders in childhood and adolescence are associated with a variety of negative outcomes, including psychosocial and school impairments and an increase in suicide risk [3].

The neurobiological bases of anxiety are complex, involving multiple neurotransmitters and neural circuits. One neurotransmitter system recently implicated in the activation of anxiety behaviors is the neuropeptide substance P (SP). Stressful stimuli can trigger the in vivo release of SP in brain areas that are known to play roles in mediating anxiety behavior, such as the amygdala, hippocampus, periaqueductal gray, nucleus accumbens, and lateral septum [4] [5]. The influences of SP are mediated by its binding to the neurokinin-1 (NK1) receptor, the distribution pattern of which mainly overlaps with the locations of SP release itself [5] [6]. Microinjections of SP into the dorsal periaqueductal gray (dPAG), a major output station for the defense reaction, have significant anxiogenic effects [7].

The demonstration of SP-related anxiogenesis prompted studies of the therapeutic value of blocking its NK-1 receptor in animal models of anxiety. In rodents, fear behaviors manifest physiologically as freezing, ultrasonic vocalizations, and a reluctance to explore new environments [8]. NK1 receptor "knockout" mouse mutants show reductions in both depressive and anxious behaviors [9]. In neonatal guinea pigs, the SP antagonist GR73632 was noted to reduce distress vocalizations induced by an SP agonist [10]. In adult male rats, Spantide, a specific NK-1 receptor antagonist, at a dose of 100 pmol, reduced freezing and escape responses after the dPAG was electrically stimulated [11]. Spantide was similarly reported to block the anxiogenic effects of SP amygdalar injections on plus maze and exploratory behavior of male rats as well as reducing freezing after dPAG stimulation, also at a dose of $100 \mathrm{pmol}$ [12].

Though the effects of the SP-NK1 pathway on anxiety behavior has been demonstrated in rodents, no studies examined whether individual differences in baseline affective temperament might interact with the pharmacological effects of blocking the NK-1 receptor. In this study, we used a rodent model useful in examining this question, ultrasonic vocalizations (USVs) after a brief maternal separation, analogous to human separation anxiety. These USVs are regarded as an indicator of the affective state of the pup (e.g. the term "distress calls" [13]), since they are reduced after the administration of anti-anxiety agents such as the benzodiazepines and neurosteroids [14]-[16]. The selective breeding of rats based on their rates of infantile ultrasonic vocalizations (USVs) has resulted in two unique lines (high and low) whose neonates reliably emit distress calls at high and low rates following a brief maternal separation [17]. The USV line difference, selected for an infantile trait, persists into adulthood. For example, heart rate in a novel environment, an indicator of stress reactivity, is significantly higher in juvenile high line rats as compared to low line rats [18]. Adult high line rats also behave more fearfully and inhibited than low line rats do; for example, high line rats take significantly longer than low line rats to emerge from a cylinder into an open field, while low line rats enter significantly more central squares and total squares in an open field than high line rats do [19].

This study examined the effects of an NK1 receptor antagonist, Spantide II, on USV rates in 7-day-old high and low line week-old pups following brief separation from the dam. USVs are a corroborated behavioral indicator of anxiety in pups [20], and infantile vocalization behavior predicts scores on independent measures of anxiety in adulthood [21]. Spantide II, an analogue of substance P that blocks SP functioning via competitive binding at the NK1 receptor sites [22], is injected into the cerebral lateral ventricles [23]. The experiment sought to characterize individual differences in the SP-NK1 pathway as well as to determine whether an anxiolytic effect of this SP antagonist would be observable in neonatal rats. 


\section{Materials and Methods}

\subsection{Subjects}

Subjects were 194N:NIH Norway infant rats bred from the $46^{\text {th }}$ generation of high and low lines in the Williams College Animal Facility, Williamstown, MA. Pregnant females were housed in clear plastic breeding cages (44 $\mathrm{cm} \times 22 \mathrm{~cm} \times 20 \mathrm{~cm}$ ) after successful mating with a same-line male, in a room with a 12-hour light-dark cycle with ad libitum access to food and water. All testing was performed during the light cycle. Experiments were performed in accordance with the National Institutes of Health Guide for Care and Use of Laboratory Animals and were approved by the Institutional Care and Use Committee. Births were noted on postnatal day 0. Litters smaller than 6 or larger than 10 pups were not used.

\subsection{Behavioral Testing}

On postnatal day 7 , the entire litter was brought to the testing room in a transfer cage, which was placed on a heating pad set at $32^{\circ} \mathrm{C}$ for 20 minutes. There were four experimental injections conditions: non-injected control, vehicle control, and two dose conditions of Spantide II. Four male and four female subjects were randomly selected from the litter; in some cases the litter did not have all possible subjects but for each litter, only one male and one female pup were tested per injection condition. Subjects were randomly assigned to experimental conditions and testing was also performed in a random order for condition and sex. Subjects $(n=49)$ in the vehicle condition received $2 \mu \mathrm{l}$ intracerebroventricular (ICV) injections of saline. Experimental subjects received ICV 2 $\mu l$ injections of either $10 \mathrm{pmol}(\mathrm{n}=47)$ or $100 \mathrm{pmol}(\mathrm{n}=43)$ of Spantide II obtained from Sigma Aldrich Chemical Company (St. Louis, MO). ICV injections were made using a glass Hamilton syringe (Cole Parmer, Chicago. IL) and a 30 gauge needle sharpened and beveled to a $22^{\circ}$ angle tip. The needle was inserted $2 \mathrm{~mm}$ into the lateral ventricle at $1 \mathrm{~mm}$ lateral and dorsal to bregma; at this age landmarks are easily visible through the skin covering the skull. Control subjects $(n=55)$ received the same handling but no injection.

Subjects were individually placed in a flat-bottomed glass dish, $190 \mathrm{~mm} \times 100 \mathrm{~mm}$, directly under a capacitance microphone with a mylar diaphragm (S-25 ultrasound bat detector, Ultra Sound Advice, London) suspended from a clamp. This system was set to detect signals at $45 \pm 5 \mathrm{kHz}$, and produced an audible signal in earphones worn by the experimenter, who could then manually count ultrasounds using a software counting program (OD Log). Calls were recorded for two minutes. Testing was conducted in an adjacent room under dim lighting.

\subsection{Data Analysis}

The effects of line, condition, and sex on USV number were evaluated with a three-factor Analysis of Variance (ANOVA), followed by LSD post-hoc testing where appropriate (criterion of $\mathrm{p}<0.05$ ). All analyses were conducted using IBM SPSS Statistics Version 21 (IBM Corp., Armonk, NY).

\section{Results}

There was a significant interaction between line and condition on the number of USVs emitted, $F(3,178)=$ 10.439, $\mathrm{p}<0.001$ ), as shown in Figure 1. This interaction is explained by the finding that the effect of condition was only significant in the high line subjects; no significant effects of the drug were apparent in low line subjects. Post-hoc testing revealed that high line subjects in the $10 \mathrm{pmol}$ and $100 \mathrm{pmol}$ drug conditions emitted significantly fewer USVs than non-injected control and vehicle control subjects (p's < 0.001). The two control groups did not differ from each other. Interestingly, high line subjects injected with 10 pmol Spantide II called marginally less than those injected with a 100 pmol solution of the drug $(\mathrm{p}<0.07)$.

There was a significant main effect of condition on the number of USVs, $F(3,178)=7.929$, $p<0.001$, an artifact due to the effects on the high line subjects. There was also a significant main effect of line on the number of USVs, $\mathrm{F}(1,178)=371.275, \mathrm{p}<0.001)$. Since the high line rats were bred specifically for high rates of USVs after brief maternal separation, the mean number of USVs was expected to be significantly higher; across conditions, low line subjects $(\mathrm{N}=80)$ emitted a mean of 32 calls per two minutes, while high line $(\mathrm{N}=114)$ produced a mean of 201 USVs. There was no main effect of sex, not any interaction of sex with any other factor. 


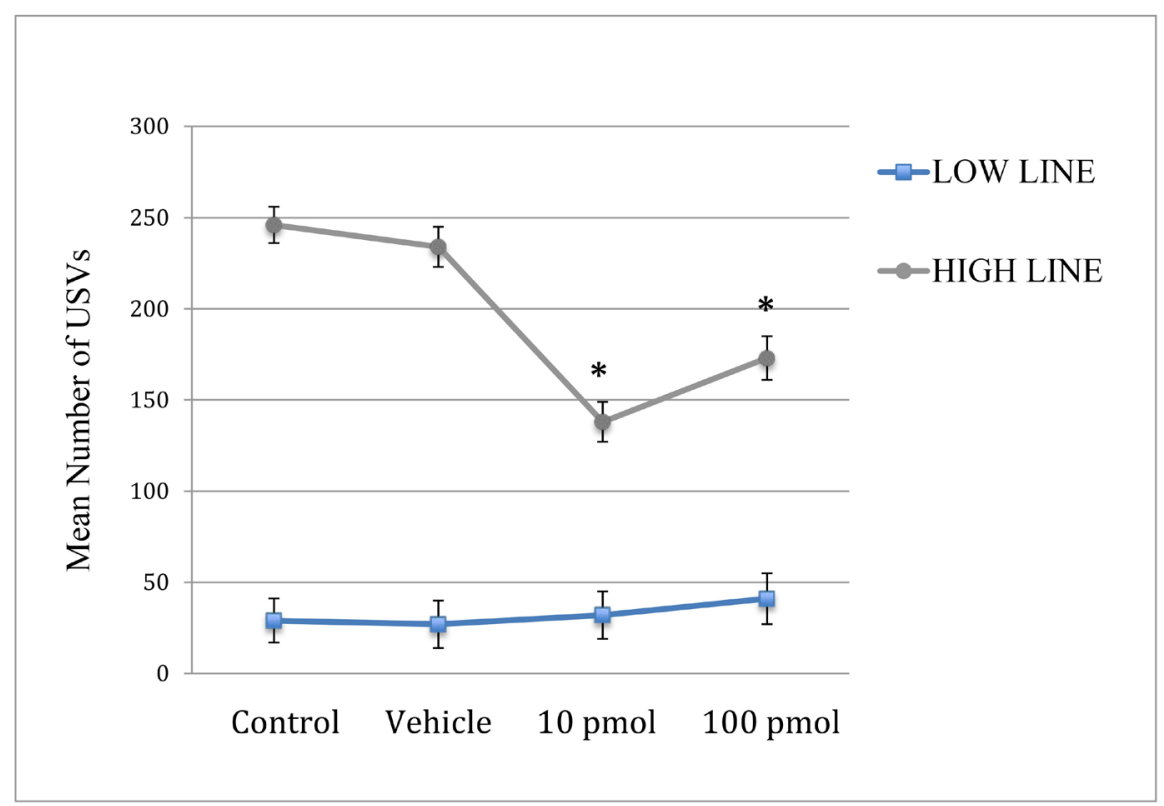

Figure 1. Mean USVs per two minutes $( \pm$ SEM) for low and high line subjects injected with Spantide II at concentrations of 10 and $100 \mathrm{pmol}$, saline vehicle, or a no-injection control. *Significantly different from both control groups (p’s < 0.001).

\section{Discussion}

A significant reduction of distress calls was observed in high line rats injected with either 10 pmol or 100 pmol Spantide II, as compared to control and vehicle groups. These results indicate that blocking NK1 receptors has an anxiolytic effect on subjects with high baseline anxiety levels. The failure of either dose of Spantide II to reduce USV rates in low line animals may be due to a line difference in density or subunit composition of NK1 receptors. Further investigation of the SP/NK1 system in this animal model might prove useful in determining this neurotransmitter system's role in stress and anxiety disorders.

The suggestion that the lower concentration $(10 \mathrm{pmol})$ was more effective than the higher dose $(100 \mathrm{pmol})$ of Spantide II parallels a similar finding in an earlier study in adult male rats. In that study, 10 pmol of Spantide II was more effective than $100 \mathrm{pmol}$ in reversing the hippocampal excitability induced by infusions of SP [24]. In this study, the mean number of USV calls in the 10 pmol drug group decreased by $43 \%$ compared to the controls, and the USV rate for the $100 \mathrm{pmol}$ group was reduced by $30 \%$. The results also extend previous research on adult male rodents to neonatal subjects of both sexes. Although no sex effects were detected, it is possible that sex effects in response to NK1 antagonists would appear after puberty.

High line rats may be a useful model system for studying stress pathologies, since humans diagnosed with anxiety disorders exhibit above-average baseline stress responses. Highlighting the interaction between baseline affective temperament and drug response emphasizes the value of matching drug treatment with individual differences in affect. The high line also offers the advantage of high rates of anxiety behavior emitted without artificial induction. While previous experiments on NK1 antagonists have used electrical stimulation of brain areas involved in defensive responses [11] [12] or infusions of SP [10], the paradigm used in this study may better correspond to environmental stressors likely to be found in the course of normal daily activities. NK1 receptor antagonists may also be useful outside the realm of anxiety, as substance P has also been implicated in modulating depression in animal models and humans [10] [25].

\section{Conclusion}

The substance P/NK1 neurotransmitter system has been demonstrated to be active in regulating anxiety in neonatal rats, which might be clinically important in developing new medications for children with anxiety disorders. The anxiolytic effect of the SP antagonist Spantide II was also found to be dependent on the subject's affective state. 


\section{Acknowledgements}

We thank the Groff Foundation for their support of this research. We thank Susan Brunelli and Myron Hofer for initiating the selective breeding of the high and low lines and their generosity in sharing this resource. We also thank Daniela Zarate for her technical support.

\section{References}

[1] Baxter, A.J., Scott, K.M., Vos, T. and Whiteford, H.A. (2012) Global Prevalence of Anxiety Disorders: A Systematic Review and Meta-Regression. Psychological Medicine, 43, 897-910. http://dx.doi.org/10.1017/S003329171200147X

[2] Merikangas, K.R., He, J.P., Burstein, M., Swanson, S.A., Avenevoli, S., Cui, L., Benjet, C., Georgiades, K. and Swendsen, J. (2010) Lifetime Prevalence of Mental Disorders in U.S. Adolescents: Results from the National Comorbidity Survey Replication-Adolescent Supplement (NCS-A). Journal of the American Academy of Child and Adolescent Psychiatry, 49, 980-989. http://dx.doi.org/10.1016/j.jaac.2010.05.017

[3] Salum, G.A., Desousa, D.A., do Rosário, M.C., Pine, D.S. and Manfro, G.G. (2013) Pediatric Anxiety Disorders: From Neuroscience to Evidence-Based Clinical Practice. Revista Brasileira de Psiquiatria, 35, S3-S21. http://dx.doi.org/10.1590/1516-4446-2013-S108

[4] Ebner, K., Muigg, P., Singewald, G. and Singewald, N. (2008) Substance P in Stress and Anxiety: NK-1 Receptor Antagonism Interacts with Key Brain Areas of the Stress Circuitry. Annals of the New York Academy of Sciences, 1144, 61-73. http://dx.doi.org/10.1196/annals.1418.018

[5] Ebner, K. and Singewald, N. (2006) The Role of Substance P in Stress and Anxiety Responses. Amino Acids, 31, 251272. http://dx.doi.org/10.1007/s00726-006-0335-9

[6] Mantyh, P.W. (2002) Neurobiology of Substance P and the NK1 Receptor. Journal of Clinical Psychiatry, 11, 6-10.

[7] De Araújo, J.E., Silva, R.C., Huston, J.P. and Brandão, M.L. (1999) Anxiogenic Effects of Substance P and Its 7-11 C Terminal, but Not the 1-7 N Terminal, Injected into the Dorsal Periaqueductal Gray. Peptides, 20, 1437-1443. http://dx.doi.org/10.1016/S0196-9781(99)00154-0

[8] Steimer, T. (2011) Animal Models of Anxiety Disorders in Rats and Mice: Some Conceptual Issues. Dialogues in Clinical Neuroscience, 13, 495-506.

[9] Bilkei-Gorzo, A. and Zimmer, A. (2005) Mutagenesis and Knockout Models: NK1 and Substance P. Handbook of Experimental Pharmacology, 169, 143-162. http://dx.doi.org/10.1007/3-540-28082-0 5

[10] Kramer M.S., Cutler, N., Feighner, J., Shrivastava, R., Carman, J., Sramek, J.J., et al. (1998) Distinct Mechanism for Antidepressant Activity by Blockade of Central Substance P Receptors. Science, 281, 1640-1645. http://dx.doi.org/10.1126/science.281.5383.1640

[11] Brenes, J.C., Broiz, A.C., Bassi, G.S., Schwarting, R.K.W. and Brandão, M.L. (2012) Involvement of Midbrain Tectum Neurokinin-Mediated Mechanisms in Fear and Anxiety. Brazilian Journal of Medical and Biological Research, 45, 349-356. http://dx.doi.org/10.1590/S0100-879X2012007500030

[12] Carvalho, C., Santos, J.M., Bassi, G.S. and Brandão, M.L. (2013) Participation of NK1 Receptors of the Amygdala on the Processing of Different Types of Fear. Neurobiology of Learning and Memory, 102, 20-27. http://dx.doi.org/10.1016/j.nlm.2013.03.004

[13] Allin, J.T. and Banks, E.M. (1972) Functional Aspects of Ultrasound Production by Infant Albino Rats (Rattus norvegicus). Animal Behavior, 20, 175-185. http://dx.doi.org/10.1016/S0003-3472(72)80189-1

[14] Carden, S.E. and Hofer, M.A. (1990) Independence of Benzodiazepine and Opiate Action in the Suppression of Isolation Distress in Rat Pups. Behavioral Neuroscience, 104, 160-166. http://dx.doi.org/10.1037/0735-7044.104.1.160

[15] Winslow, J.T. and Insel, T.R. (1991) The Infant Rat Separation Paradigm: A Novel Test for Novel Anxiolytics. Trends in Pharmacological Sciences, 12, 402-404. http://dx.doi.org/10.1016/0165-6147(91)90616-Z

[16] Zimmerberg, B., Brunelli, S.A. and Hofer, M.A. (1994) Reduction of Rat Pup Ultrasonic Vocalizations by the Neuroactive Steroid Allopregnanolone. Pharmacology, Biochemistry and Behavior, 47, 735-738. http://dx.doi.org/10.1016/0091-3057(94)90181-3

[17] Brunelli, S.A. (2005) Development and Evolution of Hidden Regulators: Selective Breeding for an Infantile Phenotype. Developmental Psychobiology, 47, 243-252. http://dx.doi.org/10.1002/dev.20090

[18] Brunelli, S.A. and Hofer, M.A. (2007) Selective Breeding for Infant Rat Separation-Induced Ultrasonic Vocalizations: Developmental Precursors of Passive and Active Coping Styles. Behavioural Brain Research, 182, 193-207. http://dx.doi.org/10.1016/j.bbr.2007.04.014

[19] Zimmerberg, B., Brunelli, S.A., Fluty, A.J. and Frye, C.A. (2005) Differences in Affective Behaviors and Hippocampal Allopregnanolone Levels in Adult Rats of Lines Selectively Bred for Infantile Vocalizations. Behavioral Brain Re- 
search, 159, 301-311. http://dx.doi.org/10.1016/j.bbr.2004.11.009

[20] Schwarting, R.K.W. and Wöhr, M. (2012) On the Relationships between Ultrasonic Calling and Anxiety-Related Behavior in Rats. Brazilian Journal of Medical and Biological Research, 45, 337-348. http://dx.doi.org/10.1590/S0100-879X2012007500038

[21] de Gomes, V., Hassan, W., Maisonnette, S., Johnson, L.R., Ramos, A. and Landeira-Fernandez, J. (2013) Behavioral Evaluation of Eight Rat Lines Selected for High and Low Anxiety-Related Responses. Behavioural Brain Research, 257, 39-48. http://dx.doi.org/10.1016/j.bbr.2013.09.028

[22] Håkanson, R., Leander, S., Asano, N., Feng, D.M. and Folkers, K. (1990) Spantide II, a Novel Tachykinin Antagonist Having High Potency and Low Histamine-Releasing Effect. Regulatory Peptides, 31, 75-82. http://dx.doi.org/10.1016/0167-0115(90)90197-5

[23] Glascock, J.J., Osman, E.Y., Coady, T.H., Rose, F.F., Shababi, M. and Lorson, C.L. (2011) Delivery of Therapeutic Agents through Intracerebroventricular (ICV) and Intravenous (IV) Injection in Mice. Journal of Visualized Experiments, 56, 2968. http://dx.doi.org/10.3791/2968

[24] Liu, H., Mazarati, A.M., Katsumori, H., Sankar, R. and Wasterlain, C.G. (1999) Substance P Is Expressed in Hippocampal Principal Neurons during Status Epilepticus and Plays a Critical Role in the Maintenance of Status Epilepticus. Proceedings of the National Academy of Sciences of the United States of America, 96, 5286-5291. http://dx.doi.org/10.1073/pnas.96.9.5286

[25] Bondy, B., Baghai, T.C., Minov, C., Schule, C., Schwarz, M.J., Zwanzger, P., et al. (2003) Substance P Serum Levels Are Increased in Major Depression: Preliminary Results. Biological Psychiatry, 53, 538-542. http://dx.doi.org/10.1016/S0006-3223(02)01544-5 
Scientific Research Publishing (SCIRP) is one of the largest Open Access journal publishers. It is currently publishing more than 200 open access, online, peer-reviewed journals covering a wide range of academic disciplines. SCIRP serves the worldwide academic communities and contributes to the progress and application of science with its publication.

Other selected journals from SCIRP are listed as below. Submit your manuscript to us via either submit@scirp.org or Online Submission Portal.
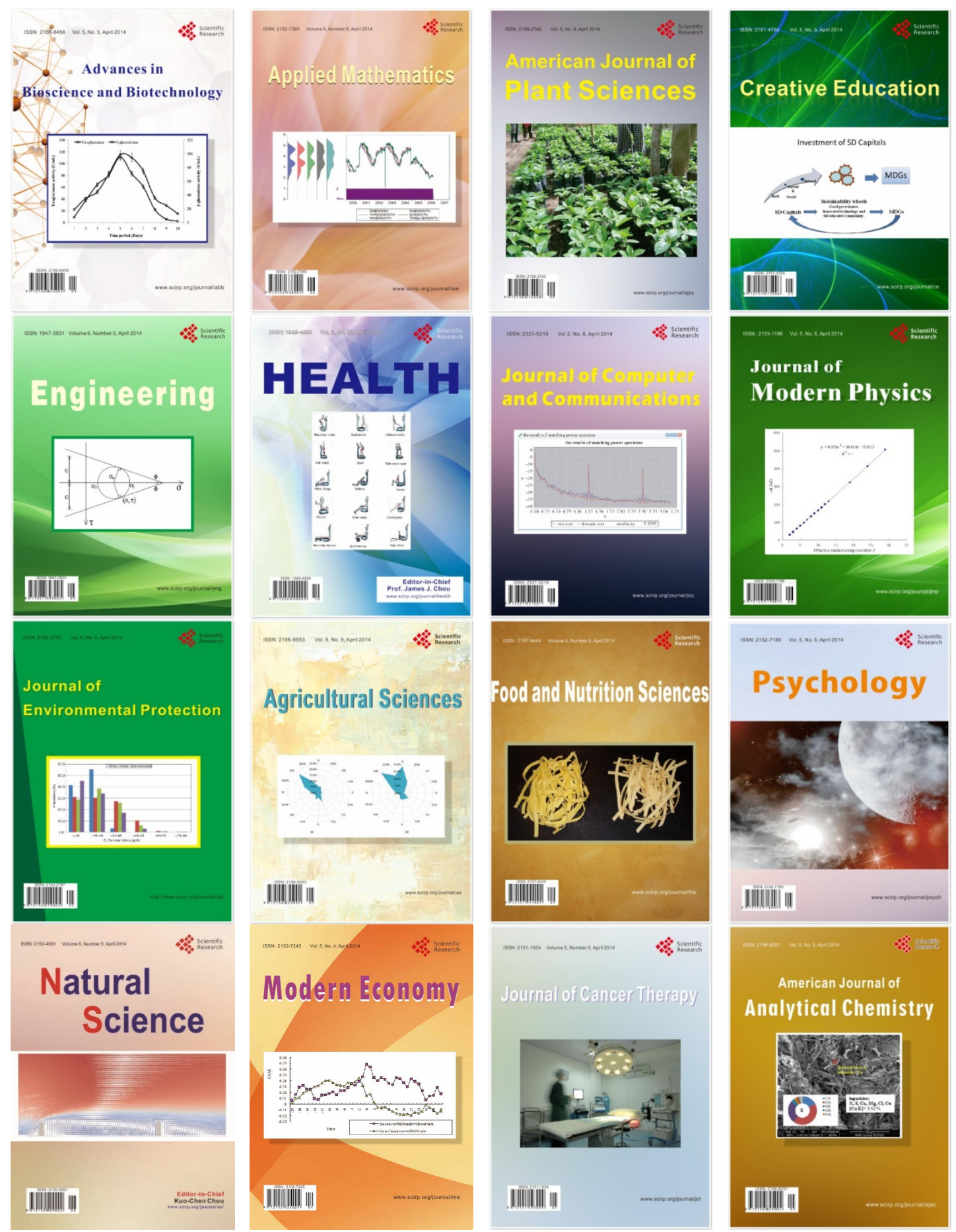